\title{
Pyogenic Vertebral Column Osteomyelitis in Adults: Analysis of Risk Factors for 30-Day and 1-Year Mortality in a Single Center Cohort Study
}

\author{
Jeevan Vettivel ${ }^{1}$, Cole Bortz ${ }^{2}$, Peter Gust Passias ${ }^{2}$, Joseph Frederick Baker ${ }^{3,4}$ \\ ${ }^{1}$ School of Medicine, University of Auckland, Auckland, New Zealand \\ ${ }^{2}$ Department of Orthopaedic Surgery, NYU Langone Medical Center, New York, NY, USA \\ ${ }^{3}$ Department of Orthopaedic Surgery, Waikato Hospital, Hamilton, New Zealand \\ ${ }^{4}$ Department of Surgery, University of Auckland, Auckland, New Zealand
}

\section{Study Design: Retrospective cohort study.}

Purpose: To describe our experience in the management and outcomes of vertebral column osteomyelitis (VCO), particularly focusing on the risk factors of early and late mortality.

Overview of Literature: Previous reports suggest a global increase in spinal column infections highlighting significant morbidity and mortality. To date, there have been no reports from our local population, and no previous report has assessed the potential relationship of frailty with mortality in a cohort of patients with VCO.

Methods: We reviewed 76 consecutive patients with VCO between 2009 and 2016 in Waikato Hospital, New Zealand. Demographic, clinical, microbiological, and treatment data were collected. Comorbidities were noted to calculate the modified Frailty Index (mFI). Mortality at 30 days and 1 year was recorded. Univariate and multivariate analyses were used to identify the predictors of mortality. Results: The mean age of patients was 64.1 years, with $77.6 \%$ being male. Most patients presented with axial back pain (71.1\%), with the lumbar spine most commonly affected $(46 \%)$. A mean of 2.1 vertebral bodies was involved. Methicillin-sensitive Staphylococcus aureus was the most common organism of infection (35.5\%), and $15.8 \%$ of patients exhibited polymicrobial infection. Twenty patients (26.3\%) underwent surgical intervention, which was more likely in patients with concomitant spinal epidural abscess (odds ratio $[\mathrm{OR}], 4.88$ ) or spondylodiscitis $(\mathrm{OR}, 3.81)$. Mortality rate was $5.2 \%$ at 30 days and $22.3 \%$ at 1 year. The presence of frailty (OR, $13.62)$ and chronic renal failure $(O R, 13.40)$ elevated the 30-day mortality risk only in univariate analysis. An increase in age (OR, 1.07) and the number of vertebral levels $(O R, 2.30)$ elevated the 1-year mortality risk in both univariate and multivariate analyses.

Conclusions: Although the $\mathrm{mFI}$ correlated with 30-day mortality in univariate analysis, it was not a significant predictor in multivariate analysis. An increase in age and the number of levels involved elevated the 1-year mortality risk.

Keywords: Vertebral osteomyelitis; Frailty; Modified Frailty Index; Mortality; Staphylococcus aureus

Received Nov 20, 2018; Revised Dec 12, 2018; Accepted Dec 16, 2018

Corresponding author: Joseph Frederick Baker

Department of Orthopaedic Surgery, Waikato Hospital, Pembroke Street, Hamilton, New Zealand

Tel: +64-7-8398899, E-mail: joseph.f.baker@gmail.com, joe.baker@auckland.ac.nz 


\section{Introduction}

The incidence of vertebral column osteomyelitis (VCO) along with other spinal column infections, such as spinal epidural abscess (SEA) and spondylodiscitis, is on the rise globally [1]. Several reports have described this challenge and have well documented the associated morbidity [2]. The diagnosis of the disease is often delayed due to the variability of symptoms and signs and nonspecific complaints on presentation $[2,3]$. Various factors are related to the increased incidence of the disease, including the use of immunosuppressive agents, increased incidence of diabetes mellitus, and an aging population $[1,4,5]$.

VCO may present variably with axial back pain, systemic upset, or neurologic symptoms, including radiculopathy, myelopathy, and in rare cases, paralysis [2]. Destructive osseous disease may result in instability that causes delayed clinical problems and mandates surgical intervention. Many cases of VCO can be managed nonoperatively if detected early and if appropriate antimicrobial therapy is initiated; however, some studies (80\%) report surgical intervention, clearly demonstrating the variability in the disease presentation [6,7]. In some cases, VCO may present subtly and a delay in diagnosis is not uncommon, often associated with the complication of broad spectrum antimicrobial treatment being commenced without identifying the spinal pathology [3].

Several variables have been associated with the increased risk of mortality, including advanced age, elevated C-reactive protein (CRP) on admission, higher Charlson Comorbidity Index, and male gender $[1,8]$. The modified Frailty Index (mFI), as a tool to measure frailty, is used increasingly to aid patient selection as it has successfully been correlated with morbidity and mortality in other disease cohorts [9]. However, to our knowledge the $\mathrm{mFI}$ has not yet been applied to a cohort of patients with VCO.

Therefore, this study aimed to provide an initial description of VCO within our population, with special consideration given to mortality rate and examined factors associated with the increased risk of mortality with a particular focus on the mFI.

\section{Materials and Methods}

This study was approved by the Waikato Hospital Clinical Audit Support Unit (3742). Patient consent was not required for this outcomes analysis. Patients, previously treated for VCO were identified using the hospital's coding system. The search was restricted to the years where digital radiographic records were available to facilitate access to radiologic reports and images to confirm the presence of osteomyelitis. Thus, we reviewed records between 2009 and 2016 so as to allow for calculation of 1-year mortality at the time of the study. During this time period, patients with VCO were treated by four spinal surgeons with the support from the infectious disease service.

Patients were excluded if their age was $<18$ years, if the spinal column infection was a surgical site infection, if the infection complicated either epidural injection or nerve root block, or if the infection was associated with malignancy. Osteomyelitis was confirmed by clinical, radiologic, and microbiologic analyses. For culture-negative cases, the diagnosis of VCO was made if there was no history of an inflammatory process that could mimic VCO and if there was a positive response to empiric antimicrobial therapy. Radiologic criteria included bony destruction of the affected vertebra(e) evident on computed tomography, plain radiograph, or a diffuse signal change within the vertebral body on both T1- and T2-weighted magnetic resonance imaging not adjacent to another infective focus (i.e., spondylodiscitis and epidural abscess).

Medical and radiographic records were reviewed for all patients for the following variables: age, sex, comorbidities, $\mathrm{mFI}$, the presenting features of spinal disease, treatment regimen, serologic results including serum albumin, hemoglobin, creatinine, CRP, microbiologic details, the length of stay, and mortality at both 30 days and 1 year. The local death registry was consulted for the 30-day and 1 -year mortality rates.

The details of the surgical intervention were recorded. For VCO, surgical intervention in our institution was indicated when the patient failed to respond to nonoperative therapy with either uncontrolled sepsis or increasing pain, spinal instability as a result of osseous, and/or soft tissue destruction or neurological compromise. Decision making was supported by multidisciplinary input from the infectious disease service.

To assess frailty, the mFI was calculated for each patient, which is an 11-item index that divides the number of conditions or disease states present in an individual by the number considered relevant (in the case of the mFI used here, 11) (Table 1). This gives an index value between 0 and 1, with 1 representing an extremely frail individual.

All data were stored in Microsoft Excel (Microsoft 
Corp., Redmond, WA, USA) and statistical analyses were performed using XLSTAT (Addinsoft, New York, NY, USA). Simple results were reported as mean (and standard deviations) or whole number (and percentage). Pearson correlation matrices were used to identify significant associations. Univariate and multivariate logistic regression analyses were performed to determine significant associations with primary outcome measures of 30-day and 1-year mortality. Subgroup analysis was performed using the Student $t$-test, when applicable.

\section{Results}

Seventy-six patients with VCO were identified for our analyses. The mean \pm standard deviation age of patients was $64.1 \pm 15.0$ years (range, $38-91$ years), and 59 patients (77.6\%) were male. The mean documented clinical followup from the time of diagnosis was 32 weeks.

Table 1. 11 Components of the modified Frailty Index

\begin{tabular}{ll} 
No. & \multicolumn{1}{c}{ Variables of the modified Frailty Index } \\
1 & Diabetes mellitus \\
\hline 2 & Functional status 2 (not independent) \\
3 & Chronic obstructive pulmonary disease or pneumonia \\
4 & Congestive cardiac failure \\
\hline 5 & Myocardial infarction \\
6 & Previous percutaneous procedure or angina \\
\hline 7 & Medically treated hypertension \\
\hline 8 & Peripheral vascular disease \\
\hline 9 & Impaired sensorium \\
10 & Transient ischaemic attack or cerebrovascular accident \\
\hline 11 & Neurologic deficit after previous cerebrovascular accident \\
\hline
\end{tabular}

Presenting features included axial pain in 54 patients (71.1\%), radiculopathy in $12(15.8 \%)$, myelopathy in nine $(11.8 \%)$, and paraplegia or quadriplegia in three (3.9\%). Concomitant SEA was observed in 21 patients $(30 \%)$ and spondylodiscitis was observed in 55 (70\%). The lumbar region was the most commonly affected region (46\%), followed by the thoracic (25\%), cervical (18\%), and junctional regions (11\%). The mean number of involved vertebrae was $2.1(0.9)$. In most patients (80\%) multiple vertebrae were involved, including three patients with five vertebrae being involved. Noncontiguous vertebral involvement was much less common (7\%). Comorbidities included neoplasia (13.2\%), diabetes mellitus (18.4\%), chronic renal impairment (9.2\%), known intravenous drug use (3.9\%), and hepatitis $\mathrm{B} / \mathrm{C}(10.5 \%)$.

Blood culture results were positive in 37 patients (48.7\%). Tissue specimens were obtained in 40 patients, either at the time of surgical intervention or by image guidance for patients managed nonoperatively, and were positive in 30 patients (75\%) among these. Urine sample analysis results were obtained for 53 patients, and positive cultures were noted in $21 \%$ of the samples. Methicillinsensitive Staphylococcus aureus was the most common organism of infection. Details of the microbiological analysis results are shown in Table 2. In total, 12 patients (15.8\%) had polymicrobial infection, 38 had monomicrobial (50\%), and in the remaining patients no specific organism was identified. Four patients (5.2\%) were diagnosed with endocarditis.

Thirty patients (26.3\%) underwent surgical intervention. Anterior column reconstruction was performed in 12 patients (15.7\%), decompression and debridement with posterior stabilization in eight (10.5\%), and decompression alone in 10 (13.2\%). Anterior column reconstruction

Table 2. Results from microbiologic investigations

\begin{tabular}{lcccc} 
Organism & No. of patients & Blood & Tissue & Urine \\
Methicillin sensitive Staphylococcus aureus & 27 & 15 & 17 & 1 \\
\hline Escherichia coli & 7 & 3 & 2 & 3 \\
\hline Methicillin resistant Staphylococcus aureus & 6 & 4 & 1 & 0 \\
Coagulase negative staphylococcus & 5 & 0 & 1 & 0 \\
\hline Enterococcus faecalis & 4 & 3 & 0 & 0 \\
Streptococcal species & 4 & 2 & 0 & 0 \\
Staphylococcus epidermidis & 2 & 2 & 5 & 5 \\
\hline Klebsiella pneumoniae & 2 & 3 & 5 \\
\hline Other & 13 & & 5 \\
\hline
\end{tabular}


Table 3. Correlation matrices with 95\% Cls in parentheses

\begin{tabular}{|c|c|c|c|c|}
\hline \multirow{2}{*}{ Variable } & \multicolumn{2}{|l|}{ 30-Day } & \multicolumn{2}{|l|}{ 1-Year } \\
\hline & $r(95 \% \mathrm{Cl})$ & $p$-value & $r(95 \% \mathrm{Cl})$ & $p$-value \\
\hline Epidural abscess & $-0.146(-0.359$ to 0.083$)$ & 0.209 & $0.092(-0.136$ to 0.311$)$ & 0.429 \\
\hline Spondylodiscitis & $-0.118(-0.334$ to 0.110$)$ & 0.310 & $-0.021(-0.246$ to 0.205$)$ & 0.855 \\
\hline No. of levels & $-0.085(-0.304$ to 0.143$)$ & 0.466 & $0.334(0.117$ to 0.520$)$ & 0.003 \\
\hline Surgical intervention & $-0.070(-0.291$ to 0.158$)$ & 0.549 & $-0.110(-0.328$ to 0.118$)$ & 0.342 \\
\hline Age & $0.121(-0.107$ to 0.338$)$ & 0.296 & $0.286(0.064$ to 0.480$)$ & 0.012 \\
\hline Axial pain & $0.021(-0.206$ to 0.245$)$ & 0.860 & $0.064(-0.164$ to 0.285$)$ & 0.582 \\
\hline Radiculopathy & $-0.102(-0.320$ to 0.126$)$ & 0.380 & $0.201(-0.026$ to 0.408$)$ & 0.082 \\
\hline Myelopathy & $0.096(-0.132$ to 0.315$)$ & 0.409 & $-0.099(-0.317$ to 0.129$)$ & 0.395 \\
\hline Para/quadriplegia & $0.216(-0.270$ to 0.180$)$ & 0.682 & $0.216(-0.010$ to 0.421$)$ & 0.062 \\
\hline Intravenous drug abuse & $-0.048(-0.270$ to 0.180$)$ & 0.682 & $0.216(-0.010$ to 0.421$)$ & 0.062 \\
\hline Diabetes mellitus & $0.040(-0.187$ to 0.263$)$ & 0.732 & $-0.011(-0.236$ to 0.251$)$ & 0.475 \\
\hline Cancer & $-0.092(-0.311$ to 0.137$)$ & 0.431 & $0.165(-0.063$ to 0.376$)$ & 0.459 \\
\hline Chronic renal failure & 0.332 (0.116 to 0.519$)$ & 0.003 & $0.047(-0.180$ to 0.270$)$ & 0.684 \\
\hline Hepatitis B/C & $-0.081(-0.301$ to 0.147$)$ & 0.488 & $0.022(-0.205$ to 0.246$)$ & 0.853 \\
\hline Methicillin resistant Staphylococcus aureus & $-0.069(-0.290$ to 0.159$)$ & 0.554 & $0.194(-0.033$ to 0.402) & 0.093 \\
\hline Modified Frailty Index & $0.400(0.192$ to 0.574$)$ & $<0.001$ & $0.144(-0.084$ to 0.358$)$ & 0.213 \\
\hline Hemoglobin & $0.045(-0.182$ to 0.268$)$ & 0.698 & $-0.055(-0.277$ to 0.173$)$ & 0.639 \\
\hline White cell count & $-0.015(-0.240$ to 0.211$)$ & 0.896 & $-0.089(-0.308$ to 0.140$)$ & 0.446 \\
\hline Albumin & $-0.113(-0.330$ to 0.115$)$ & 0.330 & $-0.093(-0.312$ to 0.135$)$ & 0.423 \\
\hline C-reactive protein & $0.031(-0.196$ to 0.255$)$ & 0.791 & $-0.082(-0.302$ to 0.146$)$ & 0.480 \\
\hline Blood culture & $-0.112(-0.329$ to 0.117$)$ & 0.337 & $0.109(-0.119$ to 0.326$)$ & 0.349 \\
\hline
\end{tabular}

Statistically significant findings highlighted in bold.

$\mathrm{Cl}$, confidence interval.

using iliac crest autograft was performed in the subaxial cervical spine in four patients. The anterior column was either shortened (five patients) or reconstructed using an expandable cage (three patients) in the thoracic spine, all via posterolateral approach except for in one patient who underwent thoracotomy. Surgical intervention was more likely in patients with associated SEA (odds ratio [OR], 4.88; 95\% confidence interval [CI], 1.70-13.96) or spondylodiscitis (OR, 3.81; 95\% CI, 1.19-12.17).

We compared patients who underwent surgical intervention with those who did not and observed that those who underwent surgery were younger $(61.1 \pm 10.4$ years versus $66.0 \pm 17.2$ years, $p=0.17$ ) and less frail with mean mFI $(0.09 \pm 0.08$ versus $0.15 \pm 0.13, p=0.04)$ compared with those who did not undergo surgery. Those who underwent surgery had a longer mean length of hospital stay (24.1 \pm 18.0 days versus $14.8 \pm 14.8$ days, $p=0.02$ ). However, there was no difference in mortality rate at either 30 days ( $3 \%$ versus $7 \%, p=0.543)$ or 1 year $(17 \%$ versus $26 \%$, $p=0.335)$.

The mean number of comorbidities as measured for the $\mathrm{mFI}$ was $1.4 \pm 1.3$, and 24 patients had only one comorbidity (31.6\%), 21 had two (27.6\%), 15 had three (19.7\%), three had four $(4.0 \%)$, and one had five comorbidities (1.3\%).

Mortality rates were $5.2 \%$ at 30 days and $22.3 \%$ at 1 -year. The presence of chronic renal failure (CRF) $(r=0.332)$ and $\mathrm{mFI}(r=0.400)$ correlated significantly with 30-day mortality. While both CRF and the presence of frailty ( $>2$ comorbidities present on the $\mathrm{mFI}$ ) resulted in a significantly greater OR for 30-day mortality in univariate analysis (13.4 and 13.6, respectively); however, this was not the case in multivariate analysis. The number of vertebral levels involved $(r=0.334)$ and patient's age $(r=0.286)$ 
Table 4. Univariate and multivariate logistic regression analysis results for both 30-day and 1-year mortality

\begin{tabular}{|c|c|c|c|c|}
\hline & \multicolumn{2}{|c|}{ 30-Day } & \multicolumn{2}{|c|}{ 1-Year } \\
\hline & Univariate & Multivariate & Univariate & Multivariate \\
\hline Chronic renal failure & 13.40 (1.55-116.23) & $6.72(0.64-70.26)$ & - & - \\
\hline Frailty status & 13.62 (1.31-141.56) & $8.67(0.74-102.32)$ & - & - \\
\hline Age & - & - & $1.06(1.01-1.11)$ & $1.07(1.01-1.12)$ \\
\hline No. of levels & - & - & $2.21(1.22-4.00)$ & 2.30 (1.21-4.37) \\
\hline
\end{tabular}

Values are presented as odds ratio (95\% confidence interval).

correlated significantly with 1-year mortality. Involvement of both increasing age ( 0.06 years) and increasing number of levels (0.83) resulted in greater OR for 1-year mortality in both univariate and multivariate analyses. Complete details of the Pearson correlation analysis are presented in Table 3, and results of logistic regression analysis are presented in Table 4.

The mean length of hospital stay was $18.5 \pm 16.7$ days. Variables correlating with the length of stay were: presence of SEA $(r=0.270)$, the number of levels involved $(r=0.293)$, presenting with para- or quadriplegia $(r=0.243)$, undergoing surgical intervention ( $r=0.274)$, IVDA status $(r=0.255)$, and positive culture for methicillin resistant Staphylococcus aureus ( $r=0.433)$.

\section{Discussion}

We aimed to detail the characteristics of VCO within our population of 76 patients over a 7 -year period. The study patients demonstrated a diverse range of presentations. A vast male predominance was noted and was consistent with previous reports $[2,3,10]$.

The early (5.2\%) and late (22.3\%) mortality rates in patients with VCO were not dissimilar to those reported elsewhere, although we acknowledge the variability in the literature. A large cohort study by Issa et al. [1] reported an early (in-hospital) mortality rate of $1.7 \%-2.2 \%$. Loibl et al. [8] found a slightly higher in-hospital mortality of $12.4 \%$, and Courjon et al. [11] reported 1-year mortality rates at $21 \%$ in patients aged $>75$ years and $3 \%$ in those aged $<75$ years; these data demonstrate the impact of increased age on mortality risk. Also, Courjon et al. [11] linked the increased mortality risk in the elderly with a greater prevalence of neoplasia in that specific cohort. We did not replicate this finding, but we did observe that CRF, frailty, and disease severity (indicated by number of in- volved vertebral levels) were associated with an increased risk of mortality, consistent with previous reports $[11,12]$.

We showed that increased frailty, as measured by the $\mathrm{mFI}$, correlated positively with mortality at 30 days. Moreover, being classified as 'frail' resulted in an increased OR for 30-day mortality, although this was shown only in univariate analysis. This is an expected finding and further highlights the usefulness of a measuring frailty in identifying patients at risk with spinal pathology. Frailty is an easily measured index and may help clinicians in decision making and in better counseling patients and their families. Presence of comorbidity has previously been linked with increased mortality risk in VCO, but to the best of our knowledge, the $\mathrm{mFI}$ in particular has not $[1,8]$. This represents a novel utility of the $\mathrm{mFI}$, which is being increasingly used in other areas of spinal surgery to predict complications [9]. In the current study, 1-year mortality, the number of involved levels, and age were correlated; age in particular has been previously linked with an increased mortality risk [12].

Consistent with other reports and one large systematic review, Staphylococcus species were, by far, the most commonly identified organisms of infection $[7,8,13]$. In a number of patients the organism of infection could not be identified, and we considered this to be due to an earlier nonspecific antimicrobial treatment. This is a perpetual challenge in the field of spinal column infection for when clinical symptoms or signs are vague the diagnosis is delayed even when the patient is systemically unwell, and thus requires some treatment effort [3]. The incidence of bacterial endocarditis was only $5.2 \%$, which was a proportion too small to draw any definite conclusions regarding considering it as a predisposing factor within this cohort. This is a notably smaller proportion than the $14 \%-37 \%$ previously reported by Courjon et al. [11] who focused on comparing younger and older patients with VCO. This 
suggests that transesophageal echocardiography should be utilized more to increase the sensitivity for diagnosis.

Our rate of surgical intervention was not dissimilar to that reported elsewhere with $26 \%$ undergoing surgical intervention for VCO. Decision making for VCO may vary according to clinician training, but intervention can often be prompted by progressive neurological deficits or spinal instability [14]. It is reassuring that surgical intervention, when necessary, can result in significant clinical improvement despite the increase in associated surgical risk [14]. Spinal column infection is frequently a mixed phenomenon, with the infection variably involving the bone, disc, and/or the surrounding structures including the paravertebral musculature and epidural space, if the infection spreads. In our study, the presence of SEA or spondylodiscitis was much more common in the surgically treated subgroup. Therefore, we must emphasize that the presence of an associated epidural abscess mandates close clinical monitoring because it generally indicates a more aggressive spinal column infection, thereby increasing the chances of required surgical intervention.

As with any retrospective study, we acknowledge that we are at the mercy of historic record keeping and availability of data. In light of this, we have attempted to minimize the influence of such factors by restricting our search period to that during which digital radiology records were available for a proper and comprehensive review. In comparison to other reports, our cohort size is also somewhat smaller but it is the first report on spinal column infection from our geographic region and highlights the challenge faced by the modern first-world healthcare system.

\section{Conclusions}

VCO remains a disease with variable presentation that may be treated appropriately either operatively or nonoperatively. The presence of an epidural abscess or spondylodiscitis is associated with a greater likelihood of surgical intervention. The mFI correlated with early mortality in this study and may help clinicians in decision making and in better counseling patients and their families.

\section{Conflict of Interest}

No potential conflict of interest relevant to this article was reported.

\section{ORCID}

Joseph Baker: https://orcid.org/0000-0002-8518-8780

\section{Author Contributions}

JV: study design, data collection, data analysis, drafting; CB: data analysis, drafting; PP: concept, drafting, final proofing; JB: concept, study design, data analysis, drafting final proofing.

\section{References}

1. Issa K, Diebo BG, Faloon M, et al. The epidemiology of vertebral osteomyelitis in the United States from 1998 to 2013. Clin Spine Surg 2018;31:E102-8.

2. Doutchi M, Seng P, Menard A, et al. Changing trends in the epidemiology of vertebral osteomyelitis in Marseille, France. New Microbes New Infect 2015;7:1-7.

3. Jean M, Irisson JO, Gras G, et al. Diagnostic delay of pyogenic vertebral osteomyelitis and its associated factors. Scand J Rheumatol 2017;46:64-8.

4. Chong BSW, Brereton CJ, Gordon A, Davis JS. Epidemiology, microbiological diagnosis, and clinical outcomes in pyogenic vertebral osteomyelitis: a 10year retrospective cohort study. Open Forum Infect Dis 2018;5:ofy037.

5. Chang WS, Ho MW, Lin PC, et al. Clinical characteristics, treatments, and outcomes of hematogenous pyogenic vertebral osteomyelitis, 12-year experience from a tertiary hospital in central Taiwan. J Microbiol Immunol Infect 2018;51:235-42.

6. Butler JS, Shelly MJ, Timlin M, Powderly WG, O'Byrne JM. Nontuberculous pyogenic spinal infection in adults: a 12-year experience from a tertiary referral center. Spine (Phila Pa 1976) 2006;31:2695700 .

7. Weissman S, Parker RD, Siddiqui W, Dykema S, Horvath J. Vertebral osteomyelitis: retrospective review of 11 years of experience. Scand J Infect Dis 2014;46:193-9.

8. Loibl M, Stoyanov L, Doenitz C, et al. Outcome-related co-factors in 105 cases of vertebral osteomyelitis in a tertiary care hospital. Infection 2014;42:503-10.

9. Yagi M, Fujita N, Okada E, et al. Impact of frailty and comorbidities on surgical outcomes and complica- 
tions in adult spinal disorders. Spine (Phila Pa 1976) 2018;43:1259-67.

10. Sapico FL, Montgomerie JZ. Vertebral osteomyelitis. Infect Dis Clin North Am 1990;4:539-50.

11. Courjon J, Lemaignen A, Ghout I, et al. Pyogenic vertebral osteomyelitis of the elderly: characteristics and outcomes. PLoS One 2017;12:e0188470.

12. Zarrouk V, Gras J, Dubee V, et al. Increased mortality in patients aged 75 years or over with pyogenic vertebral osteomyelitis. Infect Dis (Lond) 2018;50:783-7.
13. Mylona E, Samarkos M, Kakalou E, Fanourgiakis P, Skoutelis A. Pyogenic vertebral osteomyelitis: a systematic review of clinical characteristics. Semin Arthritis Rheum 2009;39:10-7.

14. Miller JA, Achey RL, Derakhshan A, Lubelski D, Benzel EC, Mroz TE. Neurologic complications, reoperation, and clinical outcomes after surgery for vertebral osteomyelitis. Spine (Phila Pa 1976) 2016;41:E197204. 